\title{
A Dynamic Channel Assignment for Coexistence of
}

\section{ZigBee /WiFi}

\author{
Chi Tao ${ }^{1,2}$ \\ 1.Shanghai Ocean University \\ 2.Key Laboratory of Fisheries Information \\ Ministry of Agriculture \\ Shanghai, China \\ tchi@shou.edu.cn
}

\author{
Yan Haowei ${ }^{1,2}$ \\ 1.Shanghai Ocean University \\ 2.Key Laboratory of Fisheries Information \\ Ministry of Agriculture \\ Shanghai, China \\ yhwshou160503765@163.com
}

\begin{abstract}
In an Agricultural Internet of Things (AIOT) system, there are a variety of wireless communication devices working simultaneously and producing interference due to using different protocols. In this paper, the problem on coexistence of ZigBee/ WiFi in AIOT is analyzed and a dynamic channel assignment method is proposed. ZigBee network defects interference and switches channel quickly to avoid the interference of the WiFi network while packet loss rate exceeds the critical value. In order to reduce energy consumption of nodes, this paper proposes power control technology based on communication distance. Experiments show that ZigBee can basicly avoid WiFi interference.
\end{abstract}

Keywords-coexistence; AIOT; ZigBee; channel assignment; power control.

\section{INTRODUCTION}

There are a variety of wireless devices used for real-time monitoring, remote control, querying and warning in AIOT. Zigbee technology, based on the IEEE 802.15.4 standard, is a short distance technology which has been widely used in AIOT. WiFi is used for long distance transmission which based on the IEEE 802.11 standard. Since ZigBee is susceptible to interference with the same frequency, that may lead to poor data acquisition. This paper explores the coexistence of Zigbee and WiFi. So far, many experts have done a lot of researches. Kang[1] proposed an interference-aware multi-channel strategy, which is based on dynamic cluster network topology. Y. Zhang[2]choosed four channels(15、20、25and26) to communicate, and this will lead to channel congestion. R.T. $\mathrm{Xu}$ [3]proposed multi-channel in the ZigBee network based on WiFi interference. Q.Wu[4] proposed a distributed approach that the channel switching is not switched together, the nodes under interference choose channel to switch firstly, the nodes with no interference then by means of adjustment to select the channel for communication. It is a pity that there is no verification of the entire network connectivity. Y. G Peng[5] designed an EA-AODV routing algorithm to extend the network lifetime. J. Han[6] proposed the methods of HEMS which can optimize household energy and lead to energy saving by taking energy consumption and production into account.

Many researchers only considered the coexistence of Zigbee/WiFi and adopted the method of switching channel to avoid WiFi interference. However, they ignore switching channel will waste a lot of energy of node. Considering the optimization for heteriod wireless network, this paper presents a new interference detection mechanism and a channel-list for dynamic channel assignment.

\section{II . OVERVIEW OF ZIGBEE/WIFI COEXISTENCE ISSUE AND MEASURES IN AIOT}

As shown in Fig. 1, the AIOT system mainly consists of enddevices, routers, coordinators and remote computer. With the development of fine agriculture, RFID, WiFi, bluetooth, satellite remote sensing and other devices have joined in AIOT.

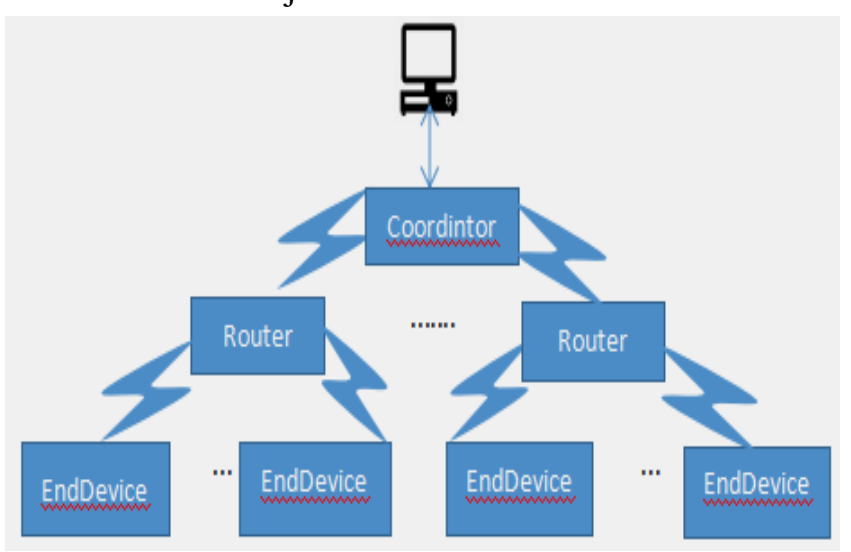

Fig. 1 System Architecture of AIOT

At present, the ZigBee technology in AIOT mainly includes the following two problems[7][8]:

\section{A. Energy Consumption.}

In the AIOT system, the energy of coordinator is supplied by power, and the energy of enddevice is powered by battery which average life is six months. This paper 
proposes that the transmit power is adjusted according to the communication distance of the equipments, which can solve the energy consumption of node well.

\section{B. Interference Problems.}

ZigBee/WiFi exist in the AIOT system and work in the ISM band of $2.4 \mathrm{GHz}$, integrating the existing attenuation model, The distance $\mathrm{R}$ between ZigBee and WiFi can be divided into three categories $(\mathrm{R} 1=35 \mathrm{~m}$; $\mathrm{R} 2=$ $80 \mathrm{~m})$ :

$\mathrm{R} 1: \mathrm{d}<\mathrm{R} 1$, ZigBee/WiFi can interfere with each other by perception.

$\mathrm{R} 2: \mathrm{R} 1<\mathrm{d}<\mathrm{R} 2$, ZigBee can be interfered by $\mathrm{WiFi}$, but not vice versa.

$\mathrm{R} 3: \mathrm{d}>\mathrm{R} 2$, the two data transmission can not be interfered with each other.

ZigBe/WiFi are working in the $2.4 \mathrm{GHz}$ ISM band as shown in Fig. 2, ZigBee works on the IEEE 802.15.4 standard and WiFi works on the IEEE 802.11 standard, part of the parameter settings are shown in TABLE I. ZigBee's bandwidth is $2 \mathrm{MHz}$, and WiFi's bandwidth is $22 \mathrm{MHz}$. ZigBee has a low transmit rate, when WiFi and ZigBee coexist, WiFi will inevitably cause serious interference to ZigBee nodes[9], which may cause network communication failure or even collapse. From Fig.1, It can be seen that the channel 15, 20, 25 and 26 are out of the communication frequency range of the $\mathrm{WiFi}$, so that these four channels are less likely to suffer from WiFi interference. And channel 11, 12, 13 and 14 are in a WiFi operating frequency range, so switching channel between the four channels is more likely to be interfered. Considering this, in this paper, when the system detects interference, the channel switches channel from the channel-list(15,20,25,26,11,16,21,12,17,22,13,18,23,14,19, 24 ).

TABLE I. The Parameter of IEEE 802.15.4/b

\begin{tabular}{|c|c|c|}
\hline Parameter & 802.15 .4 & 802.11. \\
\hline Band width & $2 \mathrm{MHz}$ & $22 \mathrm{MHz}$ \\
\hline Transmit rate & $250 \mathrm{kbps}$ & $11 \mathrm{Mbps}$ \\
\hline Transmit power & $0 \mathrm{dBm}$ & $17 \mathrm{dBm}$ \\
\hline Center Frequency & $2410 \mathrm{MHz}$ & $2412 \mathrm{MHz}$ \\
\hline ACK & No & Yes \\
\hline Transmit pattern & Every $30 \mathrm{~ms}$ & Saturated,UDP \\
\hline
\end{tabular}

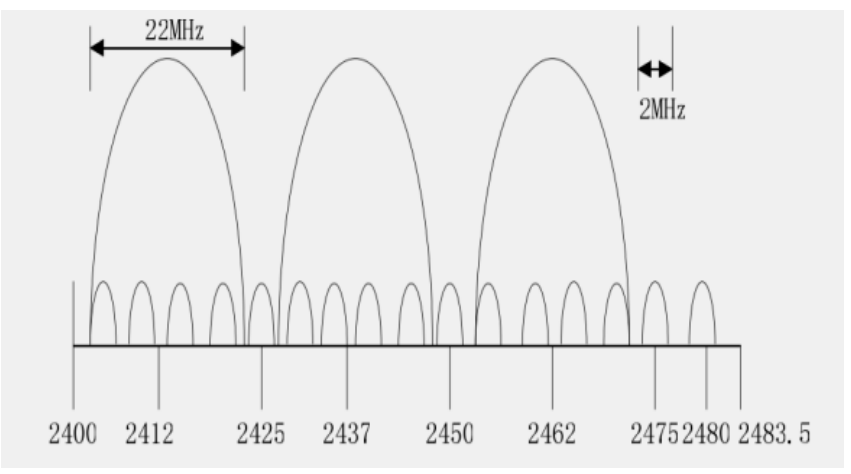

Fig. 2 WiFi/ZigBee channel distribution

\section{INTERFERENCE-AVOIDANCE ALGORITHM}

The design idea of the interference-avoidance system is: Firstly, adjusting the transmit power of nodes according to the communication distance of devices. The coordinator counts the packet loss rate of the enddevice and the router every 10 minutes. If the system detects serious WiFi interference, then the channel switches channel. The following notations will be used in our algorithm:

$\cdot R$ : communication distance

-PR: power rating

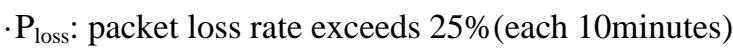

$\cdot \mathrm{P}_{\text {total: }}$ packet loss rate statistics(each 3 hours)

- $\mathrm{Ch}_{\text {start }}$ initial channel

-P: packet loss rate higher than $25 \%$ in 3 hours.

- $P_{\text {bound: }}$ critical value of packet loss rate

Adjust the transmit power rating according to the communication distance.

$$
\begin{gathered}
\text { PR } \leftrightarrow \mathrm{R} \\
P=\frac{P_{\text {loss }}}{P_{\text {total }}}
\end{gathered}
$$

If $\mathrm{P}>\mathrm{P}_{\text {bound }}$, then

$$
\mathrm{Ch}=\mathrm{Ch}_{\text {start }}
$$

If $\mathrm{Ch}_{\text {start }}$ is not free, then

$$
\mathrm{Ch}=\mathrm{Ch}+1
$$

\section{EXPERIMENTS AND RESULTS}

A. Select The Appropriate Power Based on the Communication Distance

In this paper, we adjust the nodes’ power according to the communication distance, and obtain the complete data through experiment. The process is as follows: set a ZigBee aboard as an endevice to send datas, and a ZigBee board as a coordinator to receive datas, the power range of 
the Z-Stack can be set to $-22 \mathrm{dBm} \sim+3 \mathrm{dBm}$, the experiment sets every $2 \mathrm{dBm}$ to conduct an experiment, so the power range is set to $-22 \mathrm{dBm} \sim 2 \mathrm{dBm}$. The enddevice sends data regularly, the coordinator receives data and displays information through the serial port on the computer. Fig. 3 is the result of the experiment under WiFi interference, we can see from the figure, as the power decreases, the node's communication distance decreases accordingly. Fig. 4 is the result of the experiment with no WiFi interference. From the figure, it can be seen that the same power, the communication distance of the nodes in the environment without $\mathrm{WiFi}$ interference is greatly increased, and the communication distance of node decreases as the decrease of the power. As we all know, the energy saving to the whole life in AIOT system based on ZigBee plays a crucial role, according to the specific environment and distance, we can set the appropriate power to save the energy of node. The power code as follows:

MAC_INTERNAL_API uint8 macRadioSetTxPower(uint8 txPower)

\{

halIntState_t s;

/* same as above but with no lookup table, use raw register value */

HAL_ENTER_CRITICAL_SECTION(s);

reqTxPower $=$ TxPower;

HAL_EXIT_CRITICAL_SECTION(s);

/* update the radio power setting */

macRadioUpdateTxPower();

return txPower;

\}

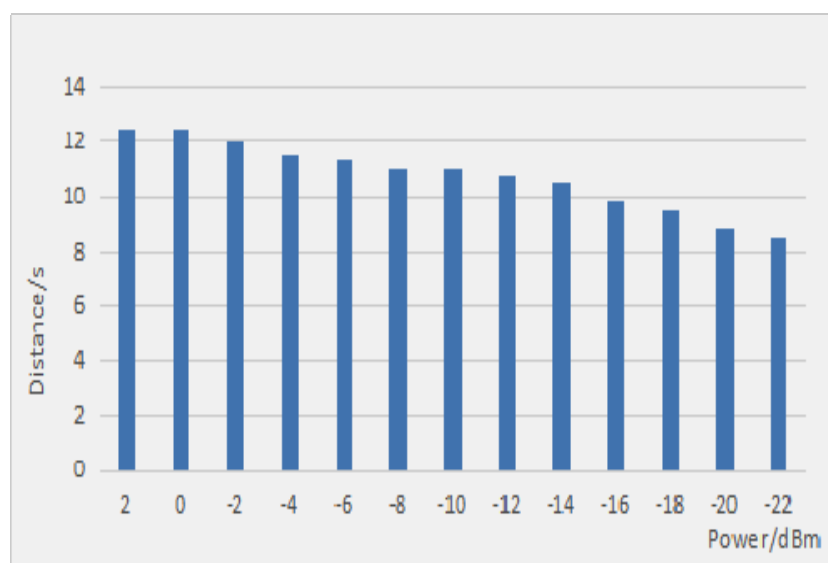

Fig.3 The Relation Between Node’s Power and Distance under WiFi

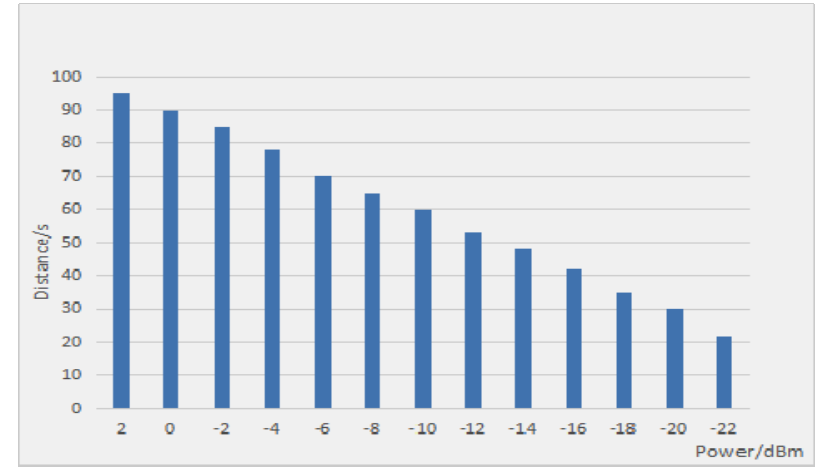

Fig. 4 The Relation Between Node's Power and Distance without WiFi

\section{B. Interference Detection Mechanism}

At present, some researchers use the RSSI detection mechanism to detect the quality of channels, a conclusion is brone out by experimrnts that RSSI value changes little in the presence of WiFi interference and no WiFi interference, so as to judge interference is not good. In this paper, the improved packet loss rate of nodes is proposed to detect the interference. The experiment uses two ZigBee boards to communicate for quality inspection, two boards have 5 meters apart. When there is no WiFi interference, the packet loss rate of two boards has been $0 \%$. When adding WiFi interference, the packet loss rate of the node is shown in Fig. 5. We can set the packet loss rate of $25 \%$ as the standard of interference, once the packet loss rate of node is higher than $25 \%$, we may identify it is being disrupted by WiFi interference.

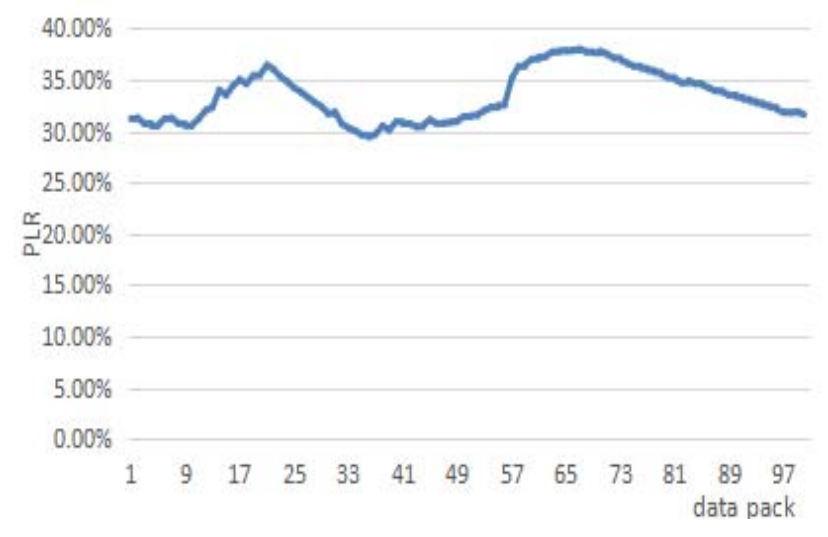

Fig.5 Packet Loss Rate of ZigBee Nodes under WiFi

\section{Dynamic Channel Assignment Technique}

The system will start scanning from channel 15. If channel 15 meets the assignment condition, the coordinator broadcasts the channel, the system will select channel the 15 to communicate. If the channel 15 does not satisfy the condition, the system will continue to select channel 20 from the channel-list to start energy scanning, repeating down until it finds a channel which meets the 
communication conditions. This avoid the traditional CSMA / CA [10] mechanism blindly scanning the channel and greatly wasting the energy of node. Experiments show that the network can communicate properly by dynamic channel assignment method.

\section{D.Network-wide Connectivity Maintenance}

Suppose that we implement a tree-like network topology, when the system detects the interference, the coordinator chooses an idle channel from channel-list and then broadcasts the communication channel to the neighbor nodes in the whole network. All nodes receive notification of switching channel, the whole network can be connected.

The experiment uses the standard ZigBee board to carry on the experiment and displays the data through the serial port on the computer. The result of packet loss rate is maintain at $0.00 \%$ by dynamic channel assignment method, which indicates that ZigBee frequency band is not affected by WiFi communication frequency band, it greatly increases the stability of the AIOT system. The results of this experiment show that this is consistent with the theoretical analysis, meeting the design of AIOT system .

\section{V . CONCLUSION AND FUTURE WORK}

In this paper, we use ZigBee boards to do a lot of experiments, the results provide a reference for the equipment disposition in AIOT, which can effectively solve the problem of coexistence between Zigbee and WiFi. Of course, for large-scale Internet of things in the industry, ZigBee nodes throw randomly, the proposed method of reducing power consumption has a certain degree of difficulty, that need we to do more in-depth study in the future.

\section{ACKNOWLEDGEMENTS}

This work is partly supported by the national natural science fund(61561027) and the shanghai natural science fund(16ZR1415100). Thanks to Key Laboratory of Fisheries Information Ministry of Agriculture.

\section{REFERENCES}

[1]Min, Suk Kang, et al. "Adaptive Interference-Aware Multi-Channel Clustering Algorithm in a ZigBee Network in the Presence of WLAN Interference." International Symposium on Wireless Pervasive Computing IEEE, 2007.

[2]Y. Zhang ,J. Wu, Y. Luo.’Design of a novel ZigBee-WiFi wireless gateway and its anti-jamming technology,” Journal of Computer Applications and Software, 2014 (5): 122-124.

[3]R.T. Xu, “Zigbee network based on multi-channel WiFi interference avoidance method,” Tianjin University, 2011.

[4]Q. Wu ,J.Y. Yuan , "Research on anti-co-channel interference based on ZigBee and WiFi networks,” Gauge Technology and Sensors, 2013 (12): 102-104.

[5]YG Peng, YL Li, ZC Lu, J Yu, "Method for Saving Energy in Zigbee Network," International Conference on Wireless Communications, 2009: $1-3$.

[6]J Han, CS Choi, WK Park, I Lee, "Smart home energy management system including renewable energy based on ZigBee and PLC," IEEE Transactions on Consumer Electronics, 2014,60 (2): 544-54.

[7]Ortmann G F, "Promoting competitiveness in South African agriculture and agribusiness: the role of institutions, “Agrekon, 2000, 39(4):367-399.

[8]S Amir, YJ Choi, "Interference coordination scheme between WiFi and Zigbee networks," Sensor Mesh \& Ad Hoc Communications \&Networks, 2012: 91-93.

[9]X. L. Dong, "WLAN and Bluetooth interference on the ZigBee," Nanjing University of Posts and Telecommunications, 2014.

[10]G Bianchi, L Fratta, M Oliveri, "Performance evaluation and enhancement of the CSMA / CA MAC protocol for 802.11 wireless LANs "IEEE International Symposium on Personal,1996, 2: 392-396. 